\title{
An analytical beam model for the evaluation of crack tip root rotations and displacements in orthotropic specimens
}

\author{
Ilaria Monetto, Roberta Massabò \\ Department of Civil, Chemical and Environmental Engineering, University of Genova, Italy \\ ilaria.monetto@unige.it, bttp://orcid.org/0000-0002-5682-9315
}

\begin{abstract}
Explicit and simple expressions for root compliance coefficients, which can be used to define root rotations and root displacements at the crack tip cross section of orthotropic cracked beams, are derived under general self-equilibrated loading conditions at the crack tip. The effects of both shear deformations and transverse elasticity are taken into account in order to accurately define displacement fields and energy release rate. The derivation builds on and extends one-dimensional formulations in the literature. The employment of the novel analytical expressions requires the determination of one a priori unknown parameter which describes the effects of the transverse elasticity and is determined through matching of well established $2 \mathrm{D}$ results in the literature. The one-dimensional model accurately reproduces crack tip effects in symmetric isotropic and orthotropic specimens; shear deformations are included in the formulation for an accurate derivation of the root displacement coefficients; the accuracy reduces in asymmetric specimens where the matching parameter becomes load dependent.
\end{abstract}

KEYwORDS. Root rotations; Root displacements; Collinear delamination; Fracture specimens; Orthotropy.

\section{OPEN ACCESS}

Citation: Monetto, I, Massabò, R., An analytical beam model for the evaluation of crack tip root rotations and displacements in orthotropic specimens, Frattura ed Integrità Strutturale, 53 (2020) 372-393.

Received: 08.05 .2020

Accepted: 25.05 .2020 Published: 01.07.2020

Copyright: (C) 2020 This is an open access article under the terms of the CC-BY 4.0, which permits unrestricted use, distribution, and reproduction in any medium, provided the original author and source are credited.

\section{INTRODUCTION}

$\mathrm{I}$ $\mathrm{n}$ many problems of fracture in beam-type structures the effects of the transverse elasticity of the intact portion of the specimen ahead of the crack tip are not negligible and must be taken into account when calculating displacements and fracture parameters. Such effects are usually associated with relative rotations and displacements at the crack tip section between the two arms of the cracked portion of the specimen. They are known in the literature as root rotations and displacements and have been the object of many studies.

A great deal of attention has been focused on modeling the root rotations, which have been shown to enter into some important fracture mechanics calculations, such as those of the J-integral and the energy release rate [1-3]. Among the others, e.g. [1-2,4-7], reference is made here to the results presented in [3] for orthotropic cracked beams subjected to 
arbitrary end loadings. They determined the compliance coefficients describing root rotations due to elementary, selfequilibrated crack tip loading systems for a wide range of thickness-wise positions of the crack and orthotropy ratios. The results obtained through rigorous $2 \mathrm{D}$ finite element analyses were validated by comparison with results obtained using other methods for different geometries in both isotropic [2] and orthotropic [8-9] materials. Recently, the accuracy of the numerical compliance coefficients has been demonstrated in [10] and by the analytical solutions in [11], for symmetric orthotropic strips, and in [12-13] for thin layers on half-planes. Root rotation coefficients can also be obtained from expressions of the energy release rate in terms of crack tip forces using the methodology proposed in [3], which has been recently applied in [10] to Double Cantilever Beam (DCB) specimens and in [14] to sandwich specimens under general end loadings. The methodology does not allow to derive root displacements.

Little attention has been placed on deriving the root displacements, which do not directly enter into the energy release rate and mode mixity and have been shown to play a role only in special problems. As examples, they need to be included in compliance-based analyses of the strain energy release rate, in the analysis of displacement controlled fracture tests, such as the wedge test, and in the analysis of problems where contact occurs along the crack surfaces, as in the inverted four point bending test. They are also important in modeling buckling and post-buckling of the detached layers using structural theories [5-6,15].

Kanninen [16] was among the firsts to include the effects of the transverse elasticity of the intact portion ahead the crack tip in the study of crack propagation and arrest in a symmetrical DCB specimen. Because of the symmetry, half of the specimen was treated as a finite length beam which is partly free and partly supported by a Winkler foundation with stiffness modulus dependent on the beam elastic and geometrical parameters. This model was firstly based on the EulerBernoulli beam theory to be used for the analysis of stationary cracks. Subsequently, the model was extended using the Timoshenko beam theory and a generalized elastic foundation depending on two parameters [17-18]. Recently Thouless [10] proposed an approach which links to the original Kanninen's [16] formulation and uses the solution of an elastic and isotropic Euler-Bernoulli beam on an elastic foundation in order to find the relationships between the compliance coefficients describing root rotations and root displacements for a symmetric DCB specimen subjected to transverse point forces. These relationships were furthermore used to derive the root displacements also for orthotropic specimens.

In this paper, a structural model for the closed form derivation of the crack tip compliance coefficients in orthotropic specimens under general end loadings, as shown in Fig. 1a, is presented. The main goal of the work is to formulate a simple but sufficiently accurate model for practical applications, which is able to provide the root displacement coefficients. The model is based on shear deformable beam theory and builds on the Kanninen's original formulation, which is here extended in order to consider new end-loading conditions. In particular, the problem of the equilibrium of a two-layered system with elastic interlayer uplift is analysed, as shown in Fig. 1b. The solution procedure previously followed by the authors to solve the problem of multi-layered systems with imperfect interfaces [19-24] is employed and leads to explicit expressions for the root rotation and displacement compliance coefficients under different crack tip loading conditions.

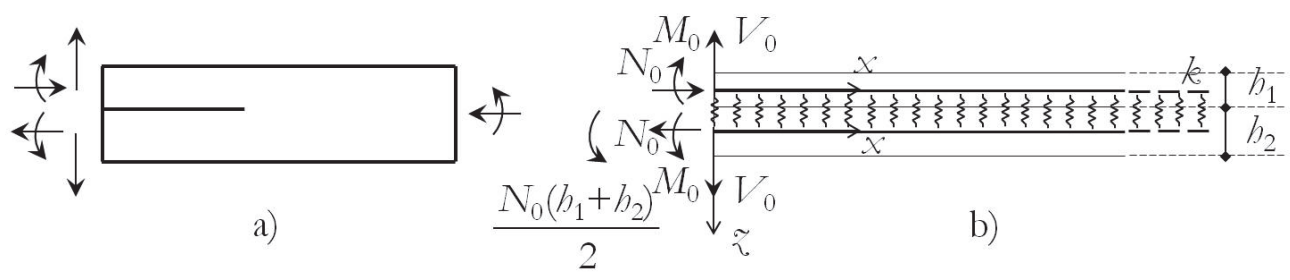

Figure 1: (a) Edge cracked specimen subjected to end loadings. (b) Elastically connected semi-infinite beams: model geometry and selfequilibrated elementary end loading conditions.

The paper is organized as follows. In the next section the equilibrium, compatibility and constitutive equations governing the model formulation are detailed and rearranged into two coupled linear differential equations for the shear and normal interfacial tractions. Details on the solution procedure leading to exact expressions for the interfacial tractions and all other static and kinematic variables are provided. In the third section explicit expressions for the relative rotation and displacement compliance coefficients under three elementary end loading conditions are derived analytically and presented for Timoshenko and Euler-Bernoulli layers. The simplified formulation based on Euler-Bernoulli beam theory is detailed in Appendix A. The novel expressions are employed on varying elastic moduli and geometrical parameters and the related numerical results are shown and discussed in the fourth section after validation through comparison with results in the literature. All numerical results shown are listed in table form in Appendix B, where the matching procedure is described. 
A practical application of the solutions to the analysis of an exemplary fracture specimen is shown in the fifth section, where the two formulations developed in the paper are compared to show the limits of the simplified model based on Euler-Bernoulli beam theory. The sixth section concludes the paper with a final discussion on the differences between the proposed model and others in the literature and on the limit of applicability of the present formulation.

\section{MODEL FORMULATION}

7 he two-layered system under consideration is shown in Fig. 1b and consists of two semi-infinite beams $(x \geq 0)$ which have constant rectangular cross sections, unit width and height $h_{i}(\mathrm{i}=1,2)$. The beams are made of the same linearly elastic and orthotropic material with principal material directions $x_{2} y, z$ and longitudinal Young's modulus $E_{x}$, transverse Young's modulus $E_{z}$, in plane shear modulus $G_{x z}$ and Poisson ratios $v_{x z}$ and $v_{x x}$. The beams are connected by a continuous bond which is assumed to be perfect in the longitudinal $x$-direction, while it ensures a partial composite action in the transverse $z$-direction. As a consequence, interlayer uplifts can occur at the interface, but no slips between the layers at their interface are possible. Such a connection is modeled by continuously distributed tangential and normal reactions; the latter are related to the relative deflection between the two beams according to a linearly elastic relationship with $k$ the proportionality constant. For our convenience, this connection modulus is expressed in terms of the average transverse stiffness of the beams as

$$
k=\frac{2 E_{z}}{h_{1}+h_{2}} k_{w}
$$

where an arbitrary correction factor, say $k_{w}$, is introduced to take into account that the beam model is only an approximation. In Kanninen's original formulation, where $E_{z}=E_{x}$ and $h_{1}=h_{2}$, the correction factor was assumed as $k_{w}=1$. Recently, with reference to the same Kanninen's symmetrical DCB specimen, Thouless [10] proposed alternative values for $k_{w}$ to obtain a reasonably good approximation for the energy release rate under 2D Linear Elastic Fracture Mechanics (LEFM) conditions. As detailed in the third section and Appendix B, $k_{w}$ is here determined so that the results found according to the proposed model match well-established root rotation compliance coefficients in the literature. Finally, the system is subject to the self-equilibrated loading condition shown in Fig. 1b: each beam is loaded at the end $x=0$ by point forces acting in the axial direction, $N_{0}$, and in the direction normal to the beam axis, $V_{0}$, and by a bending moment, $M_{0}$, in addition to a compensating moment, $N_{0}\left(h_{1}+h_{2}\right) / 2$, applied at the same end of the lower beam. These loading conditions are part of the elementary crack tip loadings which are used in the literature to fully describe the local crack tip fields and the energy release rate in beam-type structures subjected to arbitrary end loadings [2, 25].

Under the assumption of small strains, displacements and rotations and assuming Timoshenko beams to approximately account for the effects of the shear deformations, the problem of the equilibrium of the two-layered system under consideration is governed by three differential equations for each beam arm, with a total of eight unknowns: $p_{t}$ and $p_{n}$, respectively the shear and normal tractions between the two layers at their common interface, and $\varphi_{i}$, $w_{i}$ and $u_{i}$, respectively the rotation, the deflection along the z-direction and the axial displacement along the $x$-direction for the $i$-th beam $(i=1,2)$. The differential equations are

$$
\left\{\begin{array}{l}
E_{x} b_{i} u_{i}^{\prime \prime}+(-1)^{i-1} p_{t}=0 \\
\kappa_{s} G_{x x} h_{i}\left(w_{i}^{\prime \prime}+\varphi_{i}^{\prime}\right)+(-1)^{i-1} p_{n}=0 \\
\frac{1}{12} E_{x} b_{i}^{3} \varphi_{i}^{\prime \prime}-\kappa_{s} G_{x x} h_{i}\left(w_{i}^{\prime}+\varphi_{i}\right)+\frac{h_{i}}{2} p_{t}=0
\end{array}\right.
$$

where, hereinafter, primes denote differentiation with respect to $x$ and $\kappa_{s}=5 / 6$ is the shear correction factor. In the reference systems of Fig. 1b, the rotations are positive if counter-clockwise, whereas the deflections are positive if downward and the axial displacements are positive if rightward. Furthermore, the continuously distributed normal tractions $p_{n}$ are defined as 


$$
p_{n}=k\left(w_{2}-w_{1}\right)
$$

whereas the continuously distributed shear tractions $p_{t}$ are consistent with the assumption of perfect connection in the longitudinal $x$-direction imposing that

$$
u_{2}-u_{1}-\frac{h_{1}}{2} \varphi_{1}-\frac{h_{2}}{2} \varphi_{2}=0
$$

It is worthwhile noting that the interface condition expressed by Eqn. (4) makes the axial and bending problems of the two elastically connected semi-infinite beams coupled and it needs to be imposed in order to analyze the elementary end loading condition with $N_{0} \neq 0$ and related compensating moment, which would otherwise generate unrealistic sliding displacements at the interface in asymmetric specimens.

In order to solve the problem, it is convenient to rearrange the basic equations described above. Such a rearrangement, based on differentiating Eqn. (3) four times and Eqn. (4) three times with respect to $x$, leads to the following two coupled linear differential equations with constant coefficients for the unknowns $p_{n}$ and $p_{t}$ :

$$
\left\{\begin{array}{l}
p_{n}^{i v}-\hat{a} p_{n}^{\prime \prime}+\hat{b} p_{n}+\hat{c} p_{t}^{\prime}=0 \\
\hat{g} p_{t}^{\prime}+k^{-1} \hat{c}_{n}=0
\end{array}\right.
$$

having defined, according to Eqn. (1),

$$
\begin{aligned}
& \hat{a}=k \frac{h_{1}+h_{2}}{\kappa_{s} G_{x z} h_{1} h_{2}}=\frac{2 \lambda \eta k_{w}}{h_{1}^{2}} \frac{E_{x}}{\kappa_{s} G_{x z}}, \hat{b}=k \frac{12\left(h_{1}^{3}+h_{2}^{3}\right)}{E_{x} h_{1}^{3} h_{2}^{3}}=\frac{24 \lambda \eta k_{w}\left(\eta^{2}-\eta+1\right)}{b_{1}^{4}} \\
& \hat{c}=6 k\left(\frac{h_{2}^{2}-b_{1}^{2}}{E_{x} h_{1}^{2} h_{2}^{2}}\right)=\frac{12 \lambda k_{w}(1-\eta) \eta}{h_{1}^{3}} \text { and } \hat{g}=\frac{4\left(h_{1}+h_{2}\right)}{E_{x} h_{1} h_{2}}=\frac{4(1+\eta)}{E_{x} h_{1}}
\end{aligned}
$$

where

$$
\eta=\frac{h_{1}}{h_{2}}, \lambda=\frac{E_{z}}{E_{x}} \text { and } \rho=\sqrt{E_{x} E_{z}} /\left(2 G_{x z}\right)-\sqrt{v_{x z} v_{z x}}
$$

define relative thickness and orthotropy ratios in the layers and $E_{x} / G_{x z}=2\left(\rho / \sqrt{\lambda}+v_{x z}\right)$.

It is worthwhile noting that all the coefficients in Eqn. (6) are positive, except for the coefficient $\hat{c}$ which is positive only for $h_{2}>h_{1}$ and vanishes in the case of symmetrical specimen with a mid-thickness interface $\left(h_{1}=h_{2}\right)$. Furthermore, under the assumption of infinite shear stiffness of the layers $\hat{a}=0$ and Eqn. (5) so simplified is proved to govern the problem of the two elastically connected semi-infinite beams according to Euler-Bernoulli beam theory, for which the effects of the shear deformations are neglected. Appendix A presents this simplified formulation and a comparison with the model first proposed in [16] and recently reconsidered in [10] with reference to a DCB specimen.

The solution of the coupled differential problem (5) requires further rearrangement of the equations: firstly, the second of Eqn. (5) is solved for the first derivative of the interfacial shear tractions; secondly, this result is substituted in the first of Eqn. (5) leading to the following fourth-order linear differential equation with constant coefficients for the interfacial normal tractions

$$
p_{n}^{i v}-\hat{a} p_{n}+\hat{e} p_{n}=0
$$

with

$$
\hat{e}=\hat{b}-\hat{g}^{-1} k^{-1} \hat{c}^{2}=3 k \frac{\left(h_{1}+b_{2}\right)^{3}}{E_{x} h_{1}^{3} h_{2}^{3}}=\frac{6 \lambda k_{w} \eta(1+\eta)^{2}}{b_{1}^{4}}
$$


The general solution of the differential equation can be written in three different forms:

$$
p_{n}= \begin{cases}C_{1} \exp \left(\alpha_{1} x\right)+C_{2} \exp \left(\alpha_{2} x\right)+C_{3} \exp \left(-\alpha_{1} x\right)+C_{4} \exp \left(-\alpha_{2} x\right) & \text { for } \hat{d}>0 \\ \left(C_{1}+C_{2} x\right) \exp (\alpha x)+\left(C_{3}+C_{4} x\right) \exp (-\alpha x) & \text { for } \hat{d}=0 \\ C_{1} \exp \left(\alpha_{3} x\right) \cos \left(\alpha_{4} x\right)+C_{2} \exp \left(\alpha_{3} x\right) \sin \left(\alpha_{4} x\right)+ & \text { for } \hat{d}<0 \\ +C_{3} \exp \left(-\alpha_{3} x\right) \cos \left(\alpha_{4} x\right)+C_{4} \exp \left(-\alpha_{3} x\right) \sin \left(\alpha_{4} x\right) & \end{cases}
$$

where

$$
\begin{aligned}
& \hat{d}=\hat{a}^{2}-4 \hat{e}, \quad \alpha=\sqrt{\frac{\hat{a}}{2}} \\
& \alpha_{1}=\sqrt{\frac{\hat{a}+\sqrt{\hat{d}}}{2}}, \quad \alpha_{2}=\sqrt{\frac{\hat{a}-\sqrt{\hat{d}}}{2}}, \quad \alpha_{3}=\frac{1}{2} \sqrt{2 \sqrt{\hat{e}}+\hat{a}} \text { and } \alpha_{4}=\frac{1}{2} \sqrt{2 \sqrt{\hat{e}}-\hat{a}}
\end{aligned}
$$

with $\hat{a}$ and $\hat{e}$ given by Eqns. (6) and (8).

When the normal tractions along the interface between the beams have been obtained, closed form expressions for the shear tractions and for the internal forces and displacements for each beam arm can be derived, as detailed in what follows.

Firstly, integration of the second of Eqn. (5) gives the shear tractions along the interface between the two beams:

$$
p_{t}=-\hat{g}^{-1} k^{-1} \hat{c} \int p_{n} \mathrm{~d} x+C_{5}
$$

where for brevity, hereinafter, $\int \cdot \mathrm{d} x$ indicates integration of $\cdot$ once with respect to $x$; whereas $C_{5}$ is an additional arbitrary constant. Secondly, integration of Eqn. (2) gives, respectively, the axial forces

$$
N_{i}=E_{x} h_{i} u_{i}^{\prime}=(-1)^{i} \int p_{t} \mathrm{~d} x+C_{5+i}
$$

the shear forces

$$
T_{i}=\kappa_{s} G_{x z} h_{i}\left(w_{i}^{\prime}+\varphi_{i}\right)=(-1)^{i} \int p_{n} \mathrm{~d} x+C_{7+i}
$$

and the bending moments in the beams $(i=1,2)$

$$
M_{i}=\frac{1}{12} E_{x} h_{i}^{3} \varphi_{i}^{\prime}=\int\left(T_{i}-p_{t} \frac{h_{i}}{2}\right) \mathrm{d} x+C_{9+i}
$$

which contain six more arbitrary constants $C_{5+i}, C_{7+i}$ and $C_{9+i}(i=1,2)$. Thirdly, integration of the internal forces gives the rotations

$$
\varphi_{i}=\frac{12}{E_{x} h_{i}^{3}} \int M_{i} \mathrm{~d} x+C_{11+i}
$$

the axial displacements 


$$
u_{i}=\frac{1}{E_{x} h_{i}} \int N_{i} \mathrm{~d} x+C_{13+i}
$$

and the deflections of the beams $(i=1,2)$

$$
w_{i}=\int\left[\frac{T_{i}}{\kappa_{s} G_{x z} h_{i}}-\varphi_{i}\right] \mathrm{d} x+C_{15+i}
$$

with six more arbitrary constants $C_{11+i}, C_{13+i}$ and $C_{15+i}(i=1,2)$. From Eqn. (13a) the relative rotation between the two beam arms is given by

$$
\Delta \varphi=\varphi_{2}-\varphi_{1}
$$

whereas the relative deflection between the two beam arms follows directly from Eqn. (3)

$$
\Delta w=w_{2}-w_{1}=p_{n} / k
$$

The general solution derived above contains 17 arbitrary constants, which are evaluated using suitable boundary conditions. Seven conditions are obtained by imposing that the expressions given by Eqn. (13) for displacements and rotations satisfy the bond conditions Eqn. (4) of perfect connection in the longitudinal $x$-direction and Eqn. (3) of elastic connection in the transverse $z$-direction for all points of the interface. The prescription of free end boundary at infinity $(x \rightarrow \infty)$ gives five further conditions. These twelve conditions result in the following relations:

$$
\begin{aligned}
& C_{1}=C_{2}=C_{5}=C_{6}=C_{7}=C_{8}=C_{9}=C_{10}=C_{11}=0, C_{13}=C_{12} \\
& C_{17}=C_{16} \text { and } C_{15}=\left[2 C_{14}+C_{12}\left(b_{1}+b_{2}\right)\right] / 2
\end{aligned}
$$

Then, imposing that the static quantities equate the applied forces at the end $x=0$ yields $C_{3}$ and $C_{4}$, which enter the expressions given by Eqn. (14) for the relative rotation and deflection between the two beam arms. The remaining arbitrary constants $C_{12}, C_{14}$ and $C_{16}$ remain indeterminate but are not of interest here because they describe a rigid motion of the whole self-equilibrated two-layered system. Finally, substituting such results into the expressions given by Eqn. (14), evaluated at $x=0$, gives the end relative deflection and rotation, say $\Delta w_{0}$ and $\Delta \varphi_{0}$ respectively, between the two beams in terms of the end forces $\left(V_{0}, M_{0}\right.$ and $\left.N_{0}\right)$. The compliance coefficients describing the rotations and displacements follow directly from these relationships, as detailed below.

\section{ROOT ROTATION AND DISPLACEMENT COMPLIANCE COEFFICIENTS}

$\mathrm{T}$

he three elementary schematics of the bi-layered system shown in Fig. 2 are considered. The self-equilibrated end loading conditions shown in Fig. $1 \mathrm{~b}$ are reproduced by the superposition of these schematics. The relative rotation at $x=0$ given in Eqn. (14) defines the root rotation and is related to the end forces by

$$
\Delta \varphi_{0}=\frac{1}{E_{x} h_{1}}\left(d^{V_{0}} V_{0}+d^{M_{0}} \frac{M_{0}}{h_{1}}+d^{N_{0}} N_{0}\right)
$$

and the relative deflection at $x=0$ defines the root displacement and is given by

$$
\Delta w_{0}=\frac{1}{E_{x}}\left(c^{V_{0}} V_{0}+c^{M_{0}} \frac{M_{0}}{b_{1}}+c^{N_{0}} N_{0}\right)
$$


where $d^{K}$ and $c^{K}\left(K=V_{0}, M_{0}, N_{0}\right)$ are compliance coefficients, which depend on the relative thickness-wise position $\eta$ of the delamination, on the orthotropy ratios $\lambda$ and $\rho$, and on the correction factor $k_{w}$, as detailed in what follows.

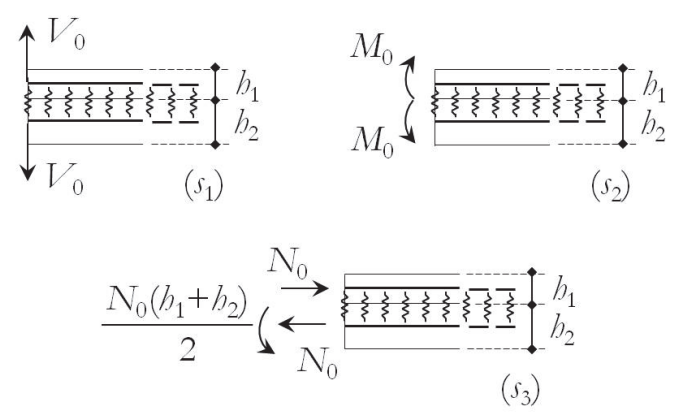

Figure 2: Schematics of the elementary loading conditions considered for the analytical derivation of the compliance coefficients.

(1) Symmetrical transverse forces

The first schematic, shown in Fig. 2(s1), corresponds to the end loading with $V_{0} \neq 0, M_{0}=0$ and $N_{0}=0$. In this case, we have

$$
d^{V_{0}}=\frac{\sqrt{3}(1+\eta)^{2}}{\sqrt{2 \eta \lambda k_{w}}}
$$

and

$$
c^{V_{0}}=(1+\eta) \sqrt{\frac{1}{\kappa_{s} \eta \lambda k_{w}} \frac{E_{x}}{G_{x z}}} F_{\alpha}
$$

with

$$
F_{\alpha}= \begin{cases}\frac{\sqrt{1+\sqrt{1-H}}+\sqrt{1-\sqrt{1-H}}}{2} & \text { for } H<1 \text { or } \hat{d}>0 \\ 1 & \text { for } H=1 \text { or } \hat{d}=0 \\ \sqrt{\frac{1+\sqrt{H}}{2}} & \text { for } H>1 \text { or } \hat{d}<0\end{cases}
$$

where

$$
H=\frac{6 \kappa_{s}^{2}(1+\eta)^{2}}{\eta \lambda k_{w}}\left(\frac{G_{x z}}{E_{x}}\right)^{2}
$$

noting that $E_{x} / G_{x z}=2\left(\rho / \sqrt{\lambda}+v_{x z}\right)$.

(2) Symmetrical bending

The second schematic, shown in Fig. 2(s2), corresponds to the end loading with $M_{0} \neq 0$ and $V_{0}=N_{0}=0$. In this case, we obtain $c^{M_{0}}=d^{V_{0}}$ defined by Eqn. (18), according to Betti's theorem, and

$$
d^{M_{0}}=(1+\eta)^{2} \sqrt{\frac{6}{\kappa_{s}} \frac{E_{x}}{G_{x z}}} F_{\alpha}
$$


together with Eqns. (20) and (21).

(3) Pair of longitudinal forces with compensating moment

The third schematic, shown in Fig. 2(s3), corresponds to the end loading with $N_{0} \neq 0, V_{0}=0$ and $M_{0}=0$. In this case, equilibrium is guaranteed by a compensating counter-clockwise moment equal to $N_{0}\left(h_{1}+h_{2}\right) / 2$ applied to the lower beam. In this case, the compliance coefficients are

$$
c^{N_{0}}=\frac{\sqrt{3}(1+\eta)^{2}}{2 \sqrt{2 \eta \lambda k_{w}}}
$$

and

$$
d^{N_{0}}=(1+\eta)^{2} \sqrt{\frac{3}{2 \kappa_{s}} \frac{E_{x}}{G_{x z}}} F_{\alpha}
$$

where $F_{\alpha}$ is defined in Eqn. (20) together with Eqn. (21).

The beam model proposed in this paper leads to the simple explicit expressions given by Eqns. (18) to (24) for the compliance coefficients describing both root rotations and root displacements as functions of the model parameters. The model parameters characterize the specimen material and geometry (namely, the orthotropy ratios and the relative thickness of the two layers), in addition to the correction factor $k_{w}$ introduced in Eqn. (1), which is the only a priori unknown model parameter.

Analogous root rotation and displacement compliance coefficients have been derived under the assumption of EulerBernoulli beam theory, as detailed in Appendix A. They are listed below:

$$
\begin{aligned}
& c_{E B}^{V_{0}}=\frac{3^{1 / 4}(1+\eta)^{3 / 2}}{2^{1 / 4}\left(\eta \lambda k_{w}^{E B}\right)^{3 / 4}}, \quad c_{E B}^{M_{0}}=d_{E B}^{V_{0}}=\frac{\sqrt{3}(1+\eta)^{2}}{\sqrt{2 \eta \lambda k_{w}^{E B}}}, \quad c_{E B}^{N_{0}}=\frac{\sqrt{3}(1+\eta)^{2}}{2 \sqrt{2 \eta \lambda k_{w}^{E B}}} \\
& d_{E B}^{M_{0}}=\frac{2^{1 / 4} 3^{3 / 4}(1+\eta)^{5 / 2}}{\left(\eta \lambda k_{w}^{E B}\right)^{1 / 4}}, \quad d_{E B}^{N_{0}}=\frac{3^{3 / 4}(1+\eta)^{5 / 2}}{2^{3 / 4}\left(\eta \lambda k_{w}^{E B}\right)^{1 / 4}}
\end{aligned}
$$

where the upper/sub-script EB has been used to differentiate with respect to the previous results. These coefficients could be obtained from Eqns. (18)-(24) by imposing infinite shear stiffness, which implies $\hat{a}=0$ and then $\hat{d}<0$.

Suitable values for $k_{w}$ or $k_{w}^{E B}$ are here chosen by reproducing well-established values for the compliance coefficients in the literature. In particular, the root rotation coefficients determined in [3] using accurate 2D finite element analyses, shown in Table 10 in appendix B, are considered. Details about the matching procedure are given in Appendix B. Tabs. 1-9 show the values of $k_{w}$ or $k_{w}^{E B}$ obtained by matching the three coefficients in isotropic and orthotropic specimens. The tables also define the values of the root rotation and displacement coefficients obtained a posteriori using the matched $k_{w}$ or $k_{w}^{E B}$ in Eqns. (18)-(24) and (25). In the next two sections some numerical results are shown and discussed, and an application of these results is presented with reference to an exemplary fracture problem.

\section{NUMERICAL RESULTS AND DISCUSSION}

$\mathrm{I}$ $\mathrm{n}$ this section, some sets of numerical results obtained by employing the analytical expressions for the root rotations and displacements derived in this paper and listed in Tabs. 1-9 are presented. This has three aims. The first is to validate the model through comparison with the results of other methods in the literature. The second is to highlight the effects of the choice of the matching compliance coefficient. The third is to discuss the effects of the geometrical and material properties of the specimen on the compliance coefficients. 


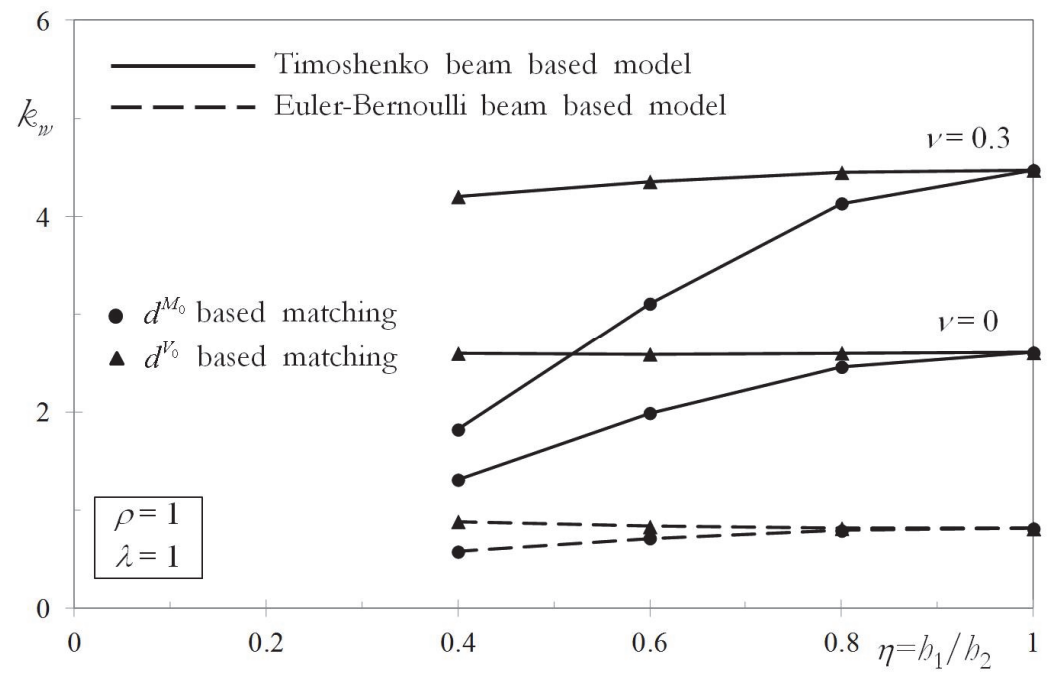

Figure 3: Correction factors of the average transverse stiffness for isotropic materials $(\rho=\lambda=1)$ with varying Poisson ratio $v=0,0.3$. Results for matching on $d^{N_{0}}$ are in Tabs. 3 and 9.

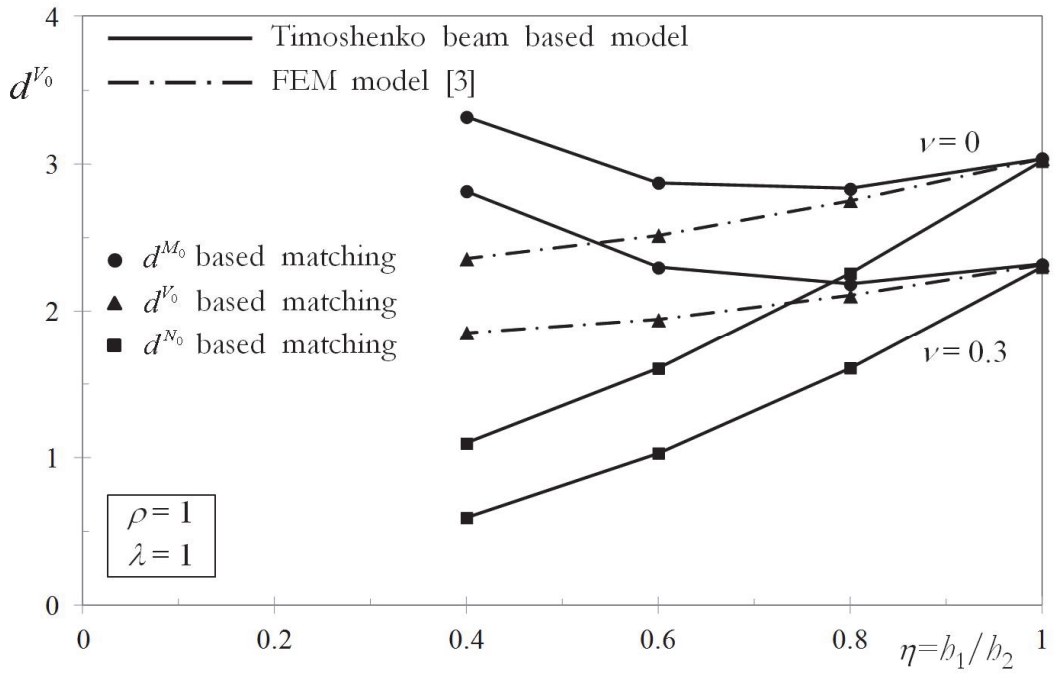

Figure 4: Relative rotation compliance coefficients under symmetrical transverse forces for isotropic materials $(\rho=\lambda=1)$ with varying Poisson ratio $v=0,0.3$.

In Figs. 3 to 8 the results based on Timoshenko beam theory (continuous lines in all figures) are shown as functions of the relative thickness-wise position $\eta$ of the delamination. Results obtained using the simplified model based on EulerBernoulli beam theory are presented in Figs. 3 and 9 to 11 (dashed lines). Fig. 3 shows the correction factors $k_{w}$ and $k_{w}^{E B}$ evaluated for isotropic materials $\left(\rho=\lambda=1\right.$ and $\left.v_{x z}=v_{z x}=v\right)$ on varying the Poisson ratio $v=0,0.3$. Figs. 4-11 show the root rotation and root displacement compliance coefficients obtained using Timoshenko and Euler-Bernoulli beam theory and the previously matched correction factors in isotropic and degenerate orthotropic specimens with $\rho=1$ and $\lambda=0.5$. The matching coefficient is shown in the diagrams using triangle marks for $d^{V_{0}}$, circle marks for $d^{M_{0}}$ and square marks for $d^{N_{0}}$; the compliance coefficients derived using 2D elasticity in [3] and used for the matching are shown by dashed-dot lines and presented in Table 10.

The diagrams, along with the equations in the previous section, highlight the features of the approach, its strengths and weaknesses. 


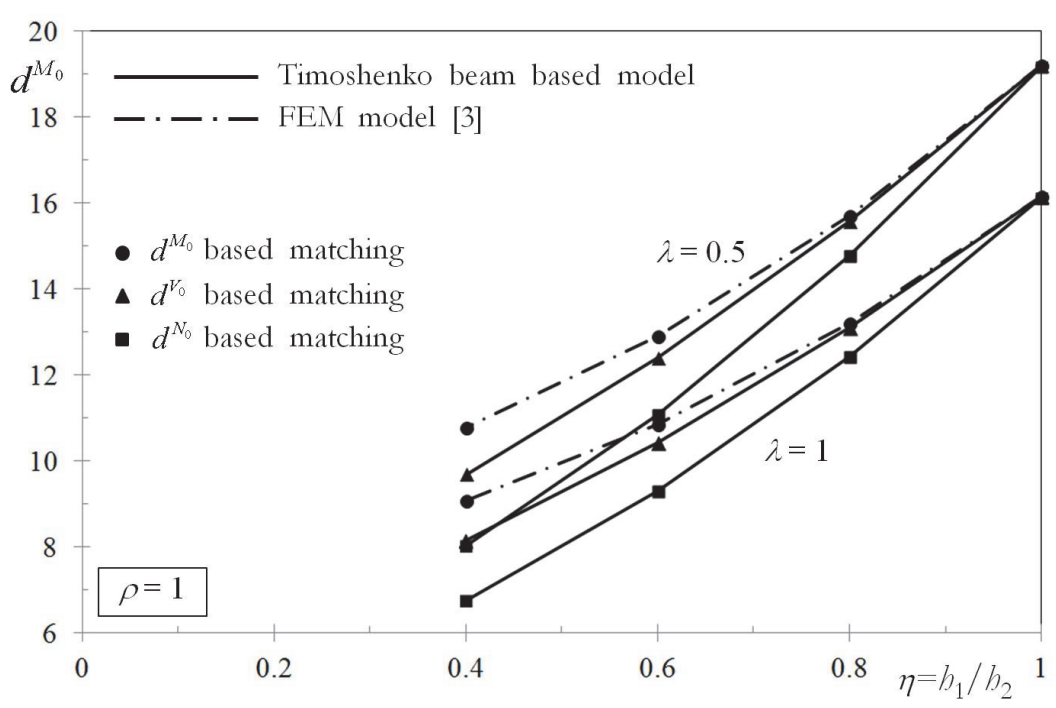

Figure 5: Relative rotation compliance coefficients under symmetrical bending for degenerate orthotropic materials $(\rho=1)$ with varying $\lambda=0.5,1$. The three upper curves refer to $\lambda=0.5$, the lower to $\lambda=1$ (isotropy).

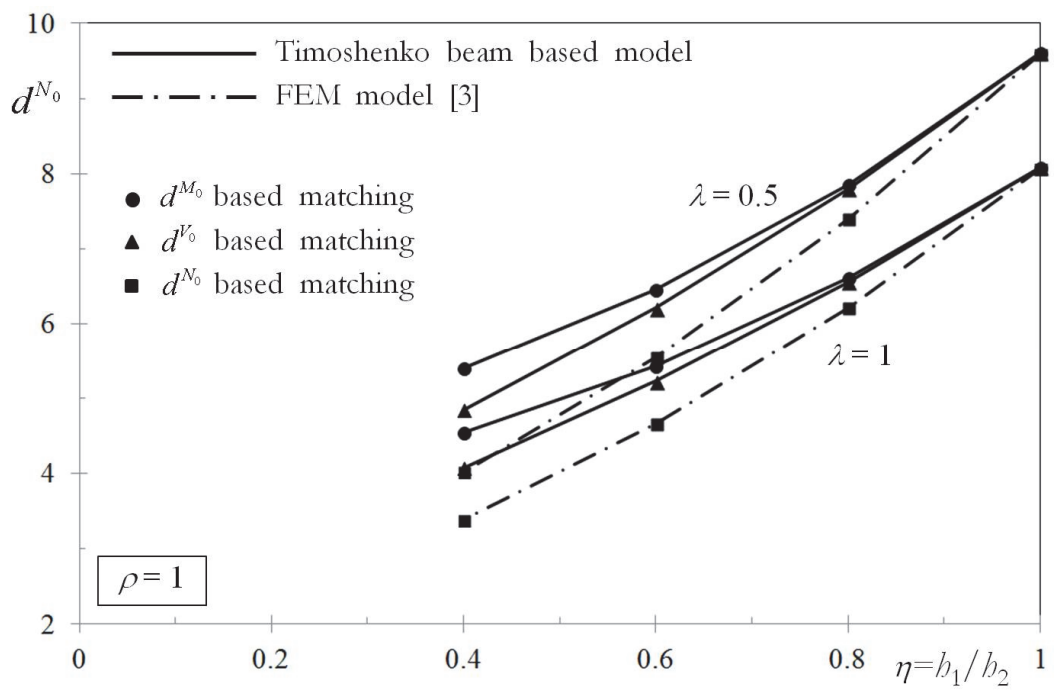

Figure 6: Relative rotation compliance coefficients under a pair of longitudinal forces for degenerate orthotropic materials $(\rho=1)$ with varying $\lambda=0.5,1$. The three upper curves refer to $\lambda=0.5$, the lower to $\lambda=1$ (isotropy).

In isotropic and orthotropic symmetric specimens, where $\eta=1$, all matching procedures lead to the same value of $k_{m}$, as shown in Fig. 3 for isotropy. The same is true for $k_{w}^{E B}$, which differs from $k_{w}$ also because it does not depend on the Poisson ratios. This implies that matching a single root rotation coefficient against the 2D solutions using Timoshenko or Euler-Bernoulli beam theory yields accurate predictions of all other root rotation coefficients, through Eqns. (18), (22), (24) and (25), as shown in Figs. 4-6 and 9. As further validation of these results, Tabs. 7 and 8 of Appendix B show that for isotropic layers $k_{w}^{E B}=0.812, d_{E B}^{V_{0}}=5.436$ and $c_{E B}^{V_{0}}=3.659$. These values perfectly match the results derived in [10] for a symmetrical DCB specimen.

In isotropic and orthotropic asymmetric specimens, with $\eta \neq 1$, using different root rotation coefficients in the matching procedure yields different predictions of $k_{w}$ or $k_{w}^{E B}$ and the difference increases on decreasing $\eta$, as shown in Fig. 3 for an isotropic layer. Fig. 3 shows that the percentage difference between the $k_{m}$ s calculated using $d^{V_{0}}$ and $d^{M_{0}}$ as matching coefficient and the Timoshenko model is about $6 \%, 7 \%$ for $\eta=0.8$ and $v=0,0.3$ and $23 \%, 29 \%$ for $\eta=0.6$ and $v=0,0.3$. The 
discrepancies are observed also in results obtained using Euler-Bernoulli beam theory and imply that the other root rotation compliance coefficients calculated a posteriori using Eqns. (18), (22), (24) and (25) differ from those of 2D elasticity in Table 10. This is shown in Figs. 4-8 and 9, which also highlight that the values of $d^{V_{0}}$ and $d^{M_{0}}$ are quite well reproduced when one of the two compliance coefficients is chosen for matching, while quite significant differences are found on the derived coefficients when matching is performed on $d^{N_{0}}$.

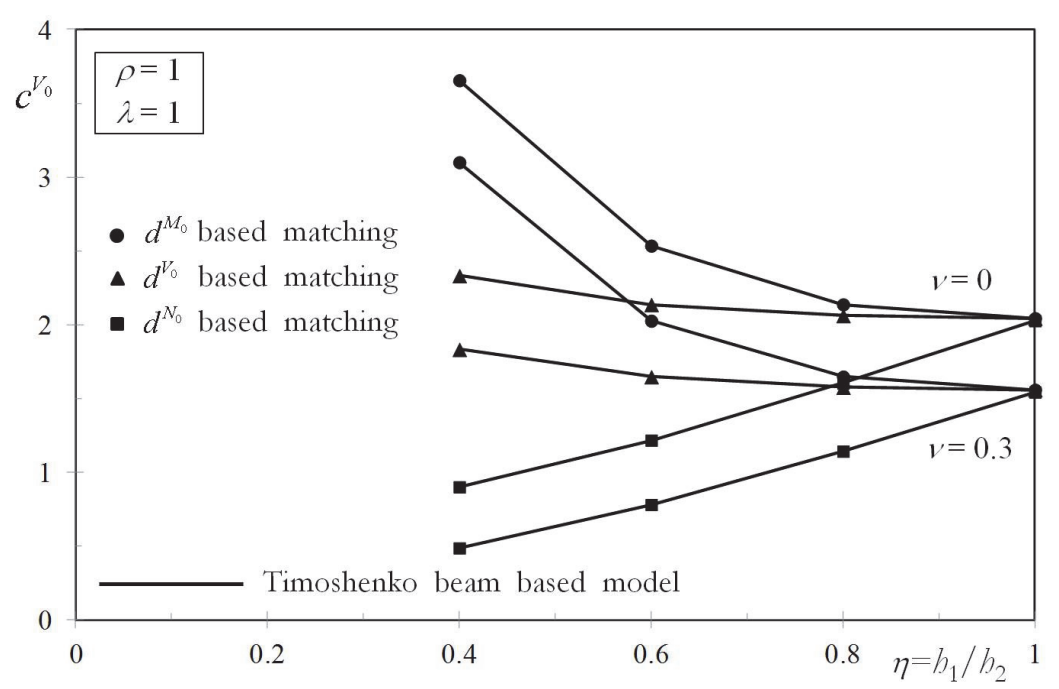

Figure 7: Relative deflection compliance coefficients under symmetrical transverse forces for isotropic materials $(\rho=\lambda=1)$ with varying Poisson ratio $v=0,0.3$.

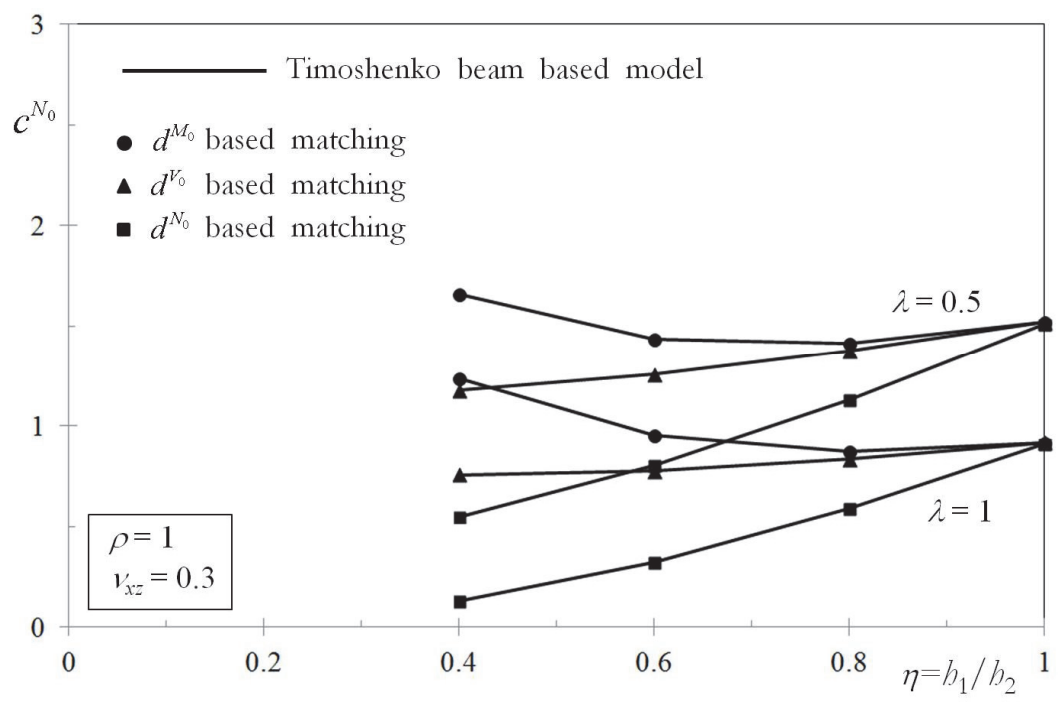

Figure 8: Relative deflection compliance coefficients under a pair of longitudinal forces for degenerate orthotropic materials $(\rho=1)$ with $v_{x z}=0.3$ and varying $\lambda=0.5,1$.

The discrepancies between the sets of results for each compliance coefficient find an explanation in the different description of the mechanical behavior according to the beam theory and $2 \mathrm{D}$ plane stress conditions. This represents a limit of applicability for the structural model proposed in the paper to specimens having a relative position of the delamination lower than 0.6. 


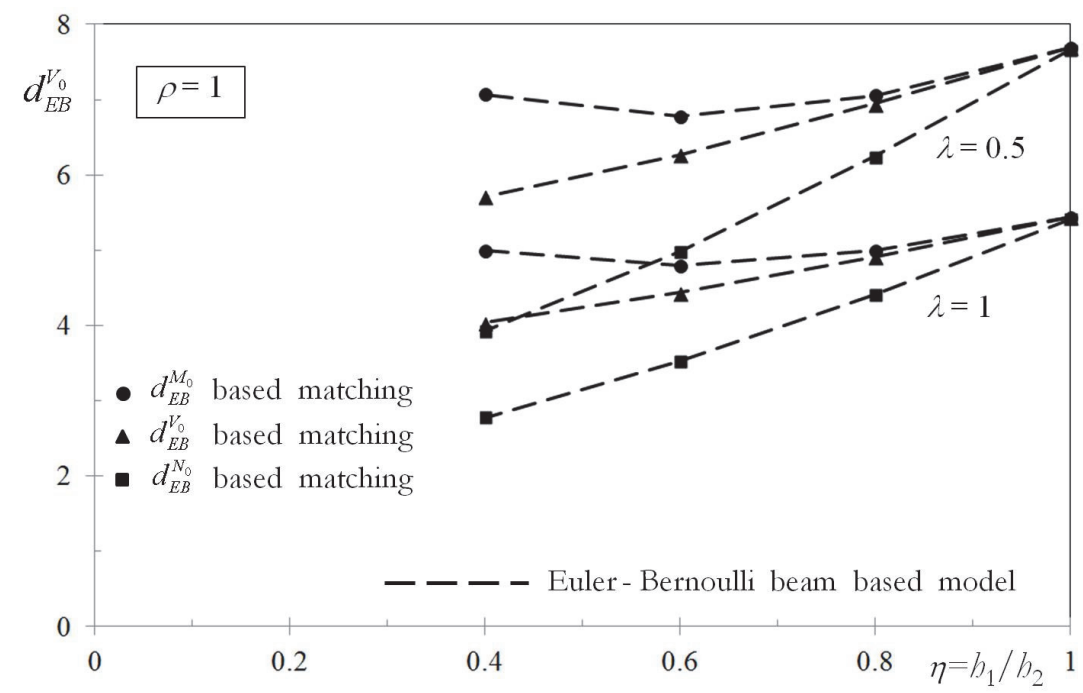

Figure 9: Relative rotation compliance coefficients under symmetrical transverse forces according to the simplified model and for degenerate orthotropic materials $(\rho=1)$ with varying $\lambda=0.5,1$.

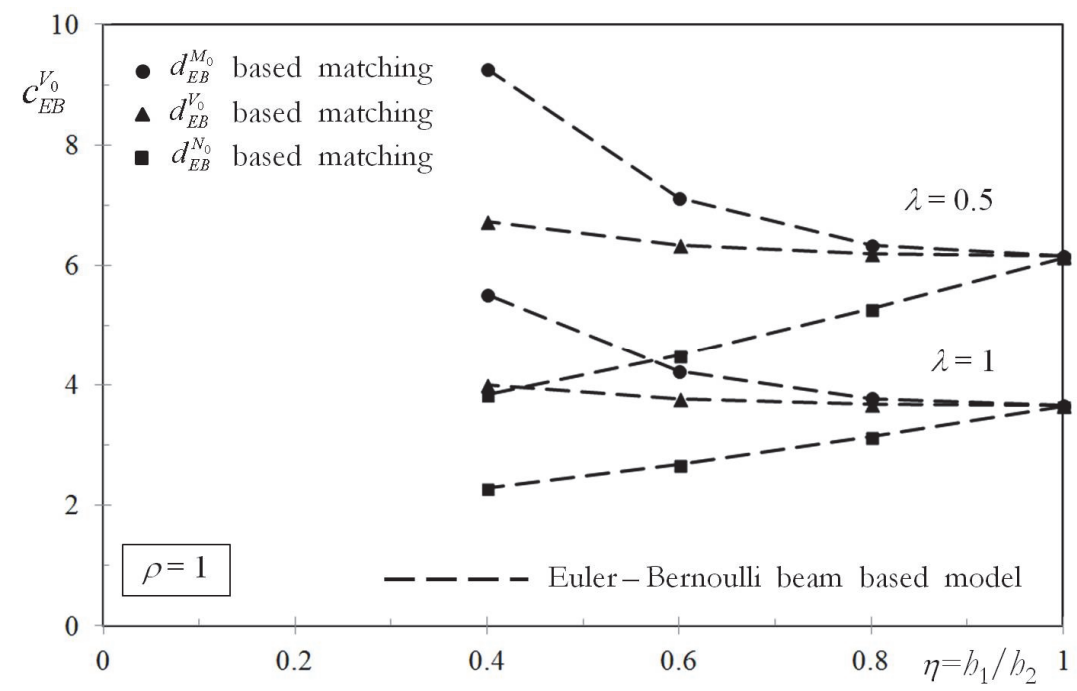

Figure 10: Relative deflection compliance coefficients under symmetrical transverse forces according to the simplified model and for degenerate orthotropic materials $(\rho=1)$ with varying $\lambda=0.5,1$.

Similar considerations can be made from the results shown in Figs. 7, 8 and 10, 11 which depict the root displacement compliance coefficients $c^{V_{0}}, c^{N_{0}}$ and $c_{E B}^{V_{0}}, c_{E B}^{N_{0}}$ under symmetrical transverse forces and a pair of longitudinal forces, respectively. $c^{M_{0}}=d^{V_{0}}$ and $c_{E B}^{M_{0}}=d_{E B}^{V_{0}}$ are shown in Figs. 4 and 9.

It is worthwhile noting that the relative rotation compliance coefficients under symmetrical bending and a pair of longitudinal forces calculated using the Euler-Bernoulli model coincide with those pertinent to the Timoshenko beam based model, shown in Figs. 5 and 6: $d^{M_{0}}=d_{E B}^{M_{0}}$ and $d^{N_{0}}=d_{E B}^{N_{0}}$, even if the correction factors differ. This is a consequence of the well known equivalence between the 2D elasticity and beam theory descriptions of the problem under such loading conditions, e.g. [8]. On the other hand, the relative rotation compliance coefficient under symmetrical transverse forces $d_{E B}^{V_{0}}$, shown in Fig. 9, differs from $d^{V_{0}}$, shown in Fig. 4, according to the following relationship 


$$
d_{E B}^{V_{0}}=d^{V_{0}}+(1+\eta) \kappa_{s}^{-1}\left(\frac{\rho}{\sqrt{\lambda}}+v_{x z}\right)
$$

which is a generalization of Eqn. (B.4) introduced for matching. The difference is made clear by referring to how the effects of shear deformations are accounted for in the two formulations. Within the Timoshenko beam theory, the effects of the shear deformations are taken into account through the beam compatibility and constitutive equations leading to Eqn. (2) which governs the problem and, as a consequence, they enter directly in the expressions of rotations and displacements. On the other hand, in the simplified formulation based on the Euler-Bernoulli beam theory the effects of shear deformations are included only in the matching procedure for the evaluation of the correction factor $k_{w}^{E B}$, as discussed in Appendix B. This point is discussed again in the next section with reference to a practical application of the two formulations to the analysis of an exemplary fracture specimen, since it has important effects on the determination of the root displacement compliance coefficients.

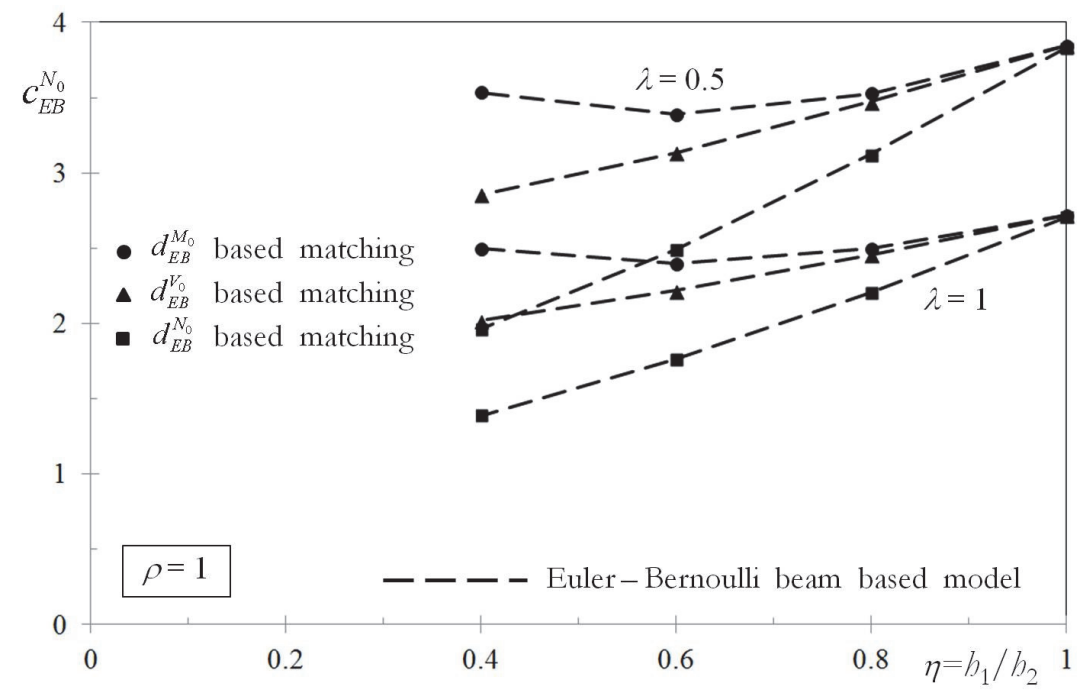

Figure 11: Relative deflection compliance coefficients under a pair of longitudinal forces according to the simplified model and for degenerate orthotropic materials $(\rho=1)$ with varying $\lambda=0.5,1$.

The final comment regards the solutions for orthotropic specimens. The correction factors obtained using the EulerBernoulli model $k_{w}^{E B}$ are independent of the orthotropy ratio $\lambda$ in all cases. This sets simple rescaling rules for the compliance coefficients in Eqn. (25) and values for $\lambda \neq 1$ can be easily obtained by rescaling the values for $\lambda=1$ by $\lambda^{-1 / 4}$ for $d_{E B}^{M_{0}}$ and $d_{E B}^{N_{0}}$, by $\lambda^{-1 / 2}$ for $d_{E B}^{V_{0}}=c_{E B}^{M_{0}}$ and $c_{E B}^{N_{0}}$ and by $\lambda^{-3 / 4} c_{E B}^{V_{0}}$. On the other hand, the correction factors obtained using the Timoshenko model depend on all the elastic constants and the rescaling rules on the compliance coefficients in Eqns. (18)-(24) are more complicated, but for $d^{M_{0}}$ and $d^{N_{0}}$ which are independent of the Poisson ratios and rescale as $\lambda^{-1 / 4}$. To conclude, all compliance coefficients depend also on the second orthotropy ratio $\rho$, however the effect of $\rho$ is only quantitative and does not modify the conclusions drawn above.

\section{APPLICATION OF THE RESULTS}

his section presents an application of the two formulations developed in the paper to the analysis of an exemplary fracture specimen. This allows to clarify how the described results can be effectively used in practice and the differences between the two formulations.

The exemplary case of the symmetric DCB specimen shown in Fig. 12a is considered. The material is linearly elastic and orthotropic with principal material directions $x, y, z$ and longitudinal Young's modulus $E_{x}$, transverse Young's modulus 
$E_{z}$, in plane shear modulus $G_{x z}$ and Poisson ratios $v_{x ₹}$ and $v_{z x}$. The specimen is subjected to a pair of transverse forces $F$ at a distance $a$ from the crack tip. The interest is here focused on the derivation of the displacement field in the specimen and the calculation of the relative transverse load point displacement $\Delta w$, in particular. In order to do this, within classical beam theories, the upper and lower arms behind the crack tip are treated as cantilever beams, built-in at the crack tip cross section $(x=a)$, and with the same length $a$ and constant rectangular cross sections with unit width and height $h$. The effective compliance of the intact portion ahead of the crack tip is accounted for through suitable root rotations and displacements.

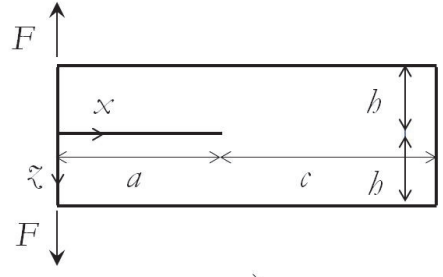

a)

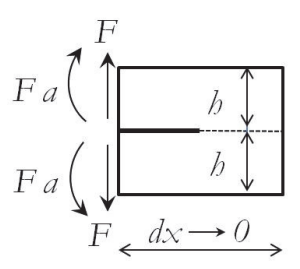

b)

Figure 12: Symmetric DCB specimen: a) geometry and loading configuration; b) loads at the crack tip.

Assuming Timoshenko beams and using the concepts of root rotations and displacements, the maximum relative deflection $\Delta w$ occuring between the two arms can be written as follows

$$
\Delta w=\frac{8 F a^{3}}{E_{x} h_{1}^{3}}+\frac{2 F a}{\kappa_{s} G_{x z} h_{1}}+a \Delta \varphi_{0}+\Delta w_{0}
$$

where the first term on the right hand side is the result of the bending moment induced by the applied loads; the second term accounts for the effects of the shear deformations along the arms; the last two terms are the corrections related to the relative rotation $\Delta \varphi_{0}$ and deflection $\Delta w_{0}$ at the crack tip cross section. Alternatively, assuming Euler-Bernoulli beams, the maximum relative deflection $\Delta w^{E B}$ at the loaded point can be written as follows

$$
\Delta w^{E B}=\frac{8 F a^{3}}{E_{x} b_{1}^{3}}+a \Delta \varphi_{0}^{E B}+\Delta w_{0}^{E B}
$$

which differs from Eqn. (27) for the absence of the term accounting for the effects of the shear deformations along the arms.

On the basis of Eqns. (16) and (17), setting the crack tip loads $N_{0}=0, V_{0}=F$ and $M_{0}=F a$, as shown in Fig. $12 \mathrm{~b}$, and noting that $E_{x} / G_{x z}=2\left(\rho / \sqrt{\lambda}+v_{x z}\right)$ yield

$$
\Delta w=\frac{8 F a^{3}}{E_{x} b_{1}^{3}}+d^{M_{0}} \frac{F a^{2}}{E_{x} h_{1}^{2}}+\frac{F a}{E_{x} b_{1}}\left[d^{V_{0}}+c^{M_{0}}+\frac{4}{\kappa_{s}}\left(\frac{\rho}{\sqrt{\lambda}}+v_{x z}\right)\right]+c^{V_{0}} \frac{F}{E_{x}}
$$

and

$$
\Delta w^{E B}=\frac{8 F a^{3}}{E_{x} h_{1}^{3}}+d_{E B}^{M_{0}} \frac{F a^{2}}{E_{x} h_{1}^{2}}+\frac{F a}{E_{x} h_{1}}\left(d_{E B}^{V_{0}}+c_{E B}^{M_{0}}\right)+c_{E B}^{V_{0}} \frac{F}{E_{x}} .
$$

Firstly, the equations above explain why some of the root displacement coefficients, those that do not multiply by the crack length, do not enter the expression of the energy release rate, which can be obtained using the compliance method. Secondly, taking into account Eqn. (26) and noting that $d^{M_{0}}=d_{E B}^{M_{0}}, c^{M_{0}}=d^{V_{0}}, c_{E B}^{M_{0}}=d_{E B}^{V_{0}}$ show that all terms of the two relations assume the same values but for the last term, which describes the effect of the root displacement due to the 
crack tip shear force. As an example, without loss of generality, in an isotropic specimen with $v=0.3$ from Tabs. 1 and 7 of Appendix B we have $c^{V_{0}}=1.559$ and $c_{E B}^{V_{0}}=3.659=2.347 c^{V_{0}}$.

In order to clarify this relevant difference and understand which model leads to the most accurate estimate for the root displacement due to crack tip shear, the finite element method has been used and a numerical analysis has been carried out through the commercial finite element code ANSYS 2019R1. A mesh consisting of plane stress four-node quadrilateral elements has been used and convergence checked by varying the mesh in size and number of elements. The corresponding root rotation and displacement compliance coefficients have been determined following the method described in [3]. The root rotation compliance coefficients due to bending moment and transverse force listed in Table 10 of Appendix B have been successfully reproduced. Furthermore, the root displacement compliance coefficient due to shear has been calculated, that is $c^{V_{0}}=1.537$. This result shows straightforwardly that the Timoshenko beam based model leads to more accurate estimates for the root displacement compliance coefficients than the Euler-Bernoulli beam based model which yields a $138 \%$ error on the prediction. In order to account for the effects of shear deformations in fracture specimens approximated as Euler-Bernoulli beams, the simplified model requires that the particular matching procedure based on Eqn. (B.4) is used, which has the effect of decreasing the correction factor of the transverse elasticity to include the effects of the shear deformations. However, this leads to accurate estimates for the root rotations but to eccessive overestimates for the root displacements.

On the other hand, it is worthwhile noting that the terms on the right hand side of Eqn. (29) have different relevance in the evaluation of the maximum relative deflection in the DCB specimen under consideration. In particular, the fourth term related to root displacements under shear is in general little to not relevant compared to the others for many geometries and orthotropy ratios. To better understand this point, the contributions due to bending moment, shear deformations and root rotations and displacements are conveniently evaluated as percentage of the total maximum relative deflection for degenerate orthotropic specimens $(\rho=1)$ with $\lambda=1,0.5,0.05,0.025$. In the case of medium/long delaminations $(a / b \geq 6)$ the term $c^{V_{0}}$ has no relevance and is smaller than $1 \%$ of the total even for very low $\lambda=0.025$. For medium/short length delaminations (e.g. $a / b=3$ ), the first term related to bending moment induced by the applied loads is the most relevant for high $\lambda=1,0.5$ but decreases from about $55 \%$ of the total for $\lambda=1$ to $26 \%$ for $\lambda=0.025$; the contribution of root rotations becomes the most relevant for low $\lambda=0.05,0.025$ and increases from $40 \%$ for $\lambda=1$ to $58 \%$ for $\lambda=0.025$; the shear deformations term increases from $5 \%$ for $\lambda=1$ to $12 \%$ for $\lambda=0.025$; once again, the contribution of $c^{V_{0}}$ has very little relevance and is smaller than $1 \%$ for $\lambda=1,0.5$ and equals to $3 \%$ for $\lambda=0.05$ and $4 \%$ for $\lambda=0.025$.

\section{CONCLUSIONS}

7 his paper deals with the analytical derivation of root compliance coefficients which can be used to define root rotations and root displacements at the crack tip cross section of orthotropic cracked beams. The work is limited to homogeneous and orthotropic materials with the axes of material symmetry parallel to the reference axes. Explicit and simple expressions have been derived for use in practical applications under general loading conditions at the crack tip. Accounting for root effects is important to accurately define energy release rate, mode mixity and displacements fields in the specimens.

The derivation builds on and extends one-dimensional formulations in the literature and describes the intact part of the specimen as two beams joined by an elastic Winkler type bond. The effects of both shear deformations and transverse elasticity are taken into account; in addition, the continuity condition imposed on the relative sliding displacements of the two beams at their interface to describe the continuity of the intact portion ahead of the crack tip makes the axial and bending problems coupled. This allows to extend Thouless' [10] treatment to problems where the geometry is not symmetrical and where root displacements may be relevant, and to loading by axial forces.

The novel analytical expressions for the root coefficients depend on the elastic constants and the geometrical parameters and one a priori unknown parameter, $k_{w}$, which defines the connection modulus and describes the effects of the transverse elasticity. The parameter is obtained through matching of well established results in the literature for the root rotation compliance coefficients.

For fixed geometry and material properties, obtaining an unique value for the parameter $k_{w}$ derived by matching the root rotations due to the different elementary loadings would imply an accuracy of the one-dimensional model in predicting the displacement field comparable to that of $2 \mathrm{D}$ elasticity. The results show that this is true in isotropic and orthotropic 
systems with mid-thickness cracks $(\eta=1)$ and in systems where the crack is only slightly misaligned, down to $\eta=0.8$. In highly asymmetric systems, the connection modulus matching the elasticity solutions appears not to be unique and depends on the elementary loading conditions. The differences are due to the limitations of the structural model, which neglects mutual coupling effects due to the deformations of adjacent points along the longitudinal axis (Winkler type support). In these problems, the matching coefficient to determine the a priori unknown model parameter should be chosen based on the role played by each compliance coefficient in the problem of interest. As an example, in a fracture mechanics problem devoted to the evaluation of the energy release rate, the fact that the contribution of the root rotations due to crack tip moments always prevails on that of the shear forces, since it varies as a higher power of the crack length, could suggest to choose the root rotation under symmetrical bending as matching coefficient.

Results also show that the model of two Timoshenko beams connected by a Winkler type bond accurately defines the root displacement coefficients of symmetric fracture specimens under general loading conditions and overcome the limitations of models based on Euler-Bernoulli beam theory, which largely overestimate predictions of these coefficients. On the other hand, the simplicity of the closed form solutions of the Euler-Bernoulli model, where the correction factor is independent of the material orthotropy and simple rescaling formulas can be used to define root coefficients, suggests the use of these solutions for all problems where the effects of the root displacements are negligible compared to the other root deformations.

\section{ACKNOWLEDGEMENTS}

7 he authors acknowledge support by the Italian Department for University and Scientific and Technological Research (MURST) in the framework of the research MIUR Prin15 project number 2015LYYXA8 "Multi-scale mechanical models for the design and optimization of micro-structured smart materials and metamaterials", coordinated by professor A. Corigliano.

RM acknowledges support by the U.S. Navy, Office of Naval Research, grant N00014-17-1-2914.

\section{REFERENCES}

[1] Wang, J., Qiao, P. (2004). Interface crack between two shear deformable elastic layers, J. Mech. Phys. Solids, 52, pp. 891-905.

[2] Li, S., Wang, J., Thouless, M.D. (2004). The effects of shear on delamination in layered materials, J. Mech. Phys. Solids, 52(1), pp. 193-214.

[3] Andrews, M.G., Massabò, R. (2007). The effects of shear and near tip deformations on energy release rate and mode mixity of edge-cracked orthotropic layers, Eng. Fracture Mech., 74, pp. 2700-2720.

[4] Pandey, R.K., Sun, C.T. (1996). Calculating strain energy release rate in cracked orthotropic beams, J. Thermoplast. Compos. Mater., 9(4), pp. 381-395.

[5] Yu, H.-H., Hutchinson, J.W. (2002). Influence of substrate compliance on buckling delamination of thin films, Int. J. Fracture, 113, pp. 39-55.

[6] Salganik, R.L., Ustinov, K.B. (2012). Deformation problem for an elastically fixed plate modeling a coating partially delaminated from the substrate (Plane Strain), Mech. Solids, 47, pp. 415-425.

[7] Ustinov, K.B. (2015). On influence of substrate compliance on delamination and buckling of coatings, Eng. Failure Analysis, 47 (PB), pp. 338-344.

[8] Suo, Z. (1990). Delamination specimens for orthotropic materials, J. Appl. Mech., 57, pp. 627-634.

[9] Brandinelli, L., Massabò, R. (2006). Mode II Weight Functions for isotropic and orthotropic Double Cantilever Beams, Int. J. Fracture, 139(1), pp. 1-25. DOI: 10.1007/s10704-006-6358-0.

[10] Thouless, M.D. (2018). Shear forces, root rotations, phase angles and delamination of layered materials, Eng. Fracture Mech., 191, pp. 153-167.

[11] Ustinov, K., Massabò, R., Lisovenko, D. (2020). Orthotropic strip with central semi-infininite crack under arbitrary loads applied far apart from the crack tip. Analytical solution, Eng. Failure Analysis, 110, 104410. DOI: 10.1016/j.engfailanal.2020.104410.

[12] Ustinov, K.B. (2019). On semi-infinite interface crack in bi-material elastic layer, European J. Mech./A Solids, 75, pp. 56-69. 
[13] Massabò, R., Ustinov, K., Barbieri, L., Berggreen, C. (2019). Fracture mechanics solutions for interfacial cracks between compressible thin layers and substrates, Coatings, 9 (3), art. no. 152. DOI: 10.3390/coatings9030152.

[14] Barbieri, L., Massabò, R., Berggreen, C. (2018). The effects of shear and near tip deformations on interface fracture of symmetric sandwich beams, Eng. Fracture Mech., 201, pp. 298-321. DOI: 10.1016/j.engfracmech.2018.06.039.

[15] Cotterell, B., Chen, Z. (2000). Buckling and cracking of thin films on compliant substrates under compression, Int. J. Fract., 104, pp. 169-79. DOI: 10.1023/A:1007628800620.

[16] Kanninen, M.F. (1973). An augmented double cantilever beam model for studying crack propagation and arrest, Int. J. Fracture, 9, pp. 83-92.

[17] Kanninen, M.F. (1974). A dynamic analysis of unstable crack propagation and arrest in the DCB test specimen, Int. J. Fracture, 10, pp. 415-430.

[18] Williams, J.G. (1989). End corrections for orthotropic DCB specimens, J. Compos. Sci. Technol., 35, pp. 367-376.

[19] Andrews, M.G., Massabò, R. (2008). Delamination in flat sheet geometries in the presence of material imperfections and thickness variations, Composites Part B, 39, pp. 139-150. DOI: 10.1016/j.compositesb.2007.02.017.

[20] Massabò, R., Cavicchi, A. (2012). Interaction effects of multiple damage mechanisms in composite sandwich beams subjected to time dependent loading, Int. J. Solids Struct., 49, pp. 720-738. DOI: 10.1016/j.ijsolstr.2011.11.012.

[21] Campi, F., Monetto, I. (2013). Analytical solutions of two-layer beams with interlayer slip and bi-linear interface law, Int. J. Solids Struct., 50, pp. 687-698.

[22] Monetto, I. (2015). Analytical solutions of three-layer beams with interlayer slip and step-wise linear interface law, Compos. Struct., 120, pp. 543-551.

[23] Monetto, I., Campi, F. (2017). Numerical analysis of two-layer beams with interlayer slip and step-wise linear interface law, Eng. Struct., 144, pp. 201-209.

[24] Monetto, I. (2019). The effects of an interlayer debond on the flexural behavior of three-layer beams, Coatings, 9 , art. no. 258 .

[25] Hutchinson, J.W., Suo, Z. (1991). Mixed mode cracking in layered materials, Adv. Appl. Mech., 29 , pp. 63-191.

\section{APPENDIX A. Simplified FORMULATION ACCORDING TO EULER-BERNOULLI BEAM THEORY}

7 his Appendix presents a simplified formulation based on modeling the two semi-infinite beams shown in Fig. 1b as linearly elastic and orthotropic Euler-Bernoulli beams.

According to Euler-Bernoulli beam theory, the shear strains within the two layers are neglected. This implies that, firstly, the rotations are related to the deflections of the beams $(i=1,2)$ as

$$
\varphi_{i}=-w_{i}^{\prime}
$$

secondly, infinite shear stiffnesses are assumed so that $\hat{a}=0$ and then $\hat{d}=-4 \hat{e}<0$. In this case, the general solution of Eqn. (7) modifies as

$$
p_{n}=\exp (\beta x)\left[C_{1} \cos (\beta x)+C_{2} \sin (\beta x)\right]+\exp (-\beta x)\left[C_{3} \cos (\beta x)+C_{4} \sin (\beta x)\right]
$$

where

$$
\beta=\sqrt[4]{\hat{e}} / \sqrt{2}
$$

with $\hat{e}$ given by Eqn. (8).

In analogy to the procedure described in the second section, the shear tractions follow from Eqn. (11), the internal forces are given by Eqn. (12), and the rotations and axial displacements are derived by Eqns. (13a-b); whereas Eqn. (13c) for the deflections is substituted by the following

$$
w_{i}=-\int \varphi_{i} \mathrm{~d} x+C_{15+i}
$$

The remaining Eqn. (14) for the relative rotation and deflection and Eqn. (15) for the arbitrary constants are still valid. Following the procedure described in the second section yields explicit expressions of the compliance coefficients 
pertinent to this simplified model based on Euler-Bernoulli beam theory. They are given by Eqn. (25). Once again, an a priori unknown parameter $k_{w}^{E B}$ enters into these expressions. Its identification requires a matching procedure similar to that presented in the third section and detailed in Appendix B also for the Timoshenko beam model.

To conclude, it is worthwhile noting that for the special case of symmetric geometry $(\eta=1)$ and loadings $\left(N_{0}=0\right)$ the expressions for the compliance coefficients $d_{E B}^{M_{0}}, d_{E B}^{V_{0}}=c_{E B}^{M_{0}}$ and $c_{E B}^{V_{0}}$ given by Eqn. (25) coincide with those reported in $[10]$ in both isotropy and orthotropy.

\section{APPENDIX B. MATCHING PROCEDURE AND SUMMARY RESULTS TABLES}

I $\mathrm{n}$ this Appendix the numerical results shown and discussed in the fourth section are listed in table form for different geometries $(\eta=1,0.8,0.6,0.4)$. The results obtained using the model according to Timoshenko beam theory are presented in Tabs. 1 to 3 for isotropic specimens $(\rho=\lambda=1)$ with varying Poisson ratios $v_{x_{2}}=v_{x x}=v=0,0.3,0.5$ and in Tabs. 4 to 6 for orthotropic specimens with $\rho=1, \lambda=0.5$ and varying Poisson ratios $v_{x_{2}}=0,0.3,0.5$. Similar results through the simplified model according to Euler-Bernoulli beam theory are reported in Tabs. 7 to 9 for $\rho=1$ and $\lambda=1$.

\begin{tabular}{|c|c|c|c|c|c|c|c|}
\hline$\eta$ & $v$ & $k_{w}$ & $d^{V_{0}}$ & $d^{M_{0}}$ & $d^{N_{0}}$ & $c^{V_{0}}$ & $c^{N_{0}}$ \\
\hline \multirow{3}{*}{1} & 0 & 2.603 & 3.036 & \multirow{3}{*}{16.154} & \multirow{3}{*}{8.077} & 2.044 & 1.518 \\
\hline & 0.3 & 4.472 & 2.316 & & & 1.559 & 1.158 \\
\hline & 0.5 & 7.116 & 1.836 & & & 1.236 & 0.918 \\
\hline \multirow{3}{*}{0.8} & 0 & 2.458 & 2.830 & \multirow{3}{*}{13.214} & \multirow{3}{*}{6.607} & 2.137 & 1.415 \\
\hline & 0.3 & 4.134 & 2.182 & & & 1.648 & 1.091 \\
\hline & 0.5 & 6.427 & 1.750 & & & 1.322 & 0.875 \\
\hline \multirow{3}{*}{0.6} & 0 & 1.988 & 2.871 & \multirow{3}{*}{51} & \multirow{3}{*}{5.426} & 2.535 & 1.436 \\
\hline & 0.3 & 3.111 & 2.295 & & & 2.027 & 1.148 \\
\hline & 0.5 & 4.486 & 1.911 & & & 1.688 & 0.956 \\
\hline \multirow{3}{*}{0.4} & 0 & 1.309 & 3.318 & \multirow{3}{*}{9.071} & \multirow{3}{*}{4.536} & 3.656 & 1.659 \\
\hline & 0.3 & 1.820 & 2.814 & & & 3.101 & 1.407 \\
\hline & 0.5 & 2.347 & 2.478 & & & 2.730 & 1.239 \\
\hline
\end{tabular}

Table 1: Numerical results according to the Timoshenko beam based model and $d^{M_{0}}$ based matching for isotropic materials $(\rho=\lambda=1)$.

\section{Matching the compliance coefficients using the Timoskenko beam based model}

In order to define the correction factor $k_{w}$, a matching has been performed on the root rotations due to moment, shear and normal force presented in [3]. The numerical values used in the present paper are the difference between the root rotation compliance coefficients for the upper, say $a_{1}^{K}$, and lower, say $a_{2}^{K}$, layers given in Table 1 of [3] (the upper-script $K=M_{0}, V_{0}, N_{0}$ denotes the end loading moment, shear or normal force). Matching is then based on equating $d^{K}=a_{1}^{K}-a_{2}^{K}$. For completeness, the numerical values $a_{1}^{K}-a_{2}^{K}$ are reported in Table 10.

As an example, with reference to the model according to the Timoshenko beam theory, for matching based on the root rotation compliance coefficient $d^{V_{0}}$, under symmetrical transverse force, the use of Eqn. (18) leads to the following expression

$$
k_{w}^{V_{0}}=\frac{3(1+\eta)^{4}}{2 \eta \lambda\left(a_{1}^{V_{0}}-a_{2}^{V_{0}}\right)^{2}}
$$

where $d^{V_{0}}=a_{1}^{V_{0}}-a_{2}^{V_{0}}$ has been enforced. Substituting Eqn. (B.1) into Eqns. (19) to (24) gives the remaining compliance coefficients. These results are reported in Table 2 for isotropic specimens and in Table 5 for degenerate orthotropic specimens with $\lambda=0.5(\rho=1)$. It is worthwhile noting that $d^{M_{0}}$ and $d^{N_{0}}$ for a generic $\lambda$ could be obtained on rescaling of $\lambda^{-1 / 4}$ those pertinent to $\lambda=1$; rescaling of the other coefficients is through more complicated expressions of the elastic 
constants. Similar considerations are valid for matching based on the other root rotation compliance coefficients $d^{M_{0}}$ and $d^{N_{0}}$, under moment and normal force, but explicit expressions for $k_{w}^{M_{0}}$ and $k_{w}^{N_{0}}$ cannot be derived from Eqns. (22) and (24) together with Eqns. (20) and (21). The pertinent numerical results are listed in Tabs. 1 and 3 for isotropic specimens and in Tabs. 4 and 6 for degenerate orthotropic specimens with $\lambda=0.5(\rho=1)$.

\begin{tabular}{|c|c|c|c|c|c|c|c|}
\hline$\eta$ & $v$ & $k_{w}$ & $d^{V_{0}}$ & $d^{M_{0}}$ & $d^{N_{0}}$ & $c^{V_{0}}$ & $c^{N_{0}}$ \\
\hline \multirow{3}{*}{1} & 0 & 2.604 & 3.036 & \multirow{3}{*}{16.153} & \multirow{3}{*}{8.077} & 2.043 & 1.518 \\
\hline & 0.3 & 4.474 & 2.316 & & & 1.559 & 1.158 \\
\hline & 0.5 & 7.120 & 1.836 & & & 1.236 & 0.918 \\
\hline \multirow{3}{*}{0.8} & 0 & 2.601 & 2.751 & \multirow{3}{*}{13.109} & \multirow{3}{*}{6.554} & 2.061 & 1.376 \\
\hline & 0.3 & 4.451 & 2.103 & & & 1.576 & 1.052 \\
\hline & 0.5 & 7.049 & 1.671 & & & 1.252 & 0.836 \\
\hline \multirow{3}{*}{0.6} & 0 & 2.590 & 2.515 & \multirow{3}{*}{10.440} & \multirow{3}{*}{5.220} & 2.137 & 1.258 \\
\hline & 0.3 & 4.358 & 1.939 & & & 1.647 & 0.970 \\
\hline & 0.5 & 6.776 & 1.555 & & & 1.321 & 0.778 \\
\hline \multirow{3}{*}{0.4} & 0 & 2.598 & 2.355 & \multirow{3}{*}{8.151} & \multirow{3}{*}{4.075} & 2.332 & 1.178 \\
\hline & 0.3 & 4.205 & 1.851 & & & 1.833 & 0.926 \\
\hline & 0.5 & 6.277 & 1.515 & & & 1.500 & 0.758 \\
\hline
\end{tabular}

Table 2: Numerical results according to the Timoshenko beam based model and $d^{V_{0}}$ based matching for isotropic materials $(\rho=\lambda=1)$.

\begin{tabular}{|c|c|c|c|c|c|c|c|}
\hline$\eta$ & $v$ & $k_{w}$ & $d^{V_{0}}$ & $d^{M_{0}}$ & $d^{N_{0}}$ & $c^{V_{0}}$ & $c^{N_{0}}$ \\
\hline \multirow{3}{*}{1} & 0 & 2.638 & 3.016 & \multirow{3}{*}{16.124} & \multirow{3}{*}{8.062} & 2.026 & 1.508 \\
\hline & 0.3 & 4.551 & 2.296 & & & 1.543 & 1.148 \\
\hline & 0.5 & 7.275 & 1.816 & & & 1.220 & 0.908 \\
\hline \multirow{3}{*}{0.8} & 0 & 3.864 & 2.257 & \multirow{3}{*}{12.} & \multirow{3}{*}{6.216} & 1.604 & 1.128 \\
\hline & 0.3 & 7.604 & 1.609 & & & 1.143 & 0.804 \\
\hline & 0.5 & 14.212 & 1.177 & & & 0.836 & 0.588 \\
\hline \multirow{3}{*}{0.6} & 0 & 6.345 & 1.607 & \multirow{3}{*}{9.310} & \multirow{3}{*}{4.655} & 1.217 & 0.803 \\
\hline & 0.3 & 15.418 & 1.031 & & & 0.781 & 0.515 \\
\hline & 0.5 & 39.156 & 0.647 & & & 0.490 & 0.323 \\
\hline \multirow{3}{*}{0.4} & 0 & 11.966 & 1.097 & \multirow{3}{*}{6.762} & \multirow{3}{*}{3.381} & 0.901 & 0.549 \\
\hline & 0.3 & 40.932 & 0.593 & & & 0.487 & 0.297 \\
\hline & 0.5 & 217.687 & 0.257 & & & 0.211 & 0.129 \\
\hline
\end{tabular}

Table 3: Numerical results according to the Timoshenko beam based model and $d^{N_{0}}$ based matching for isotropic materials $(\rho=\lambda=1)$.

\begin{tabular}{|c|c|c|c|c|c|c|c|}
\hline$\eta$ & $v$ & $k_{w}$ & $d^{V_{0}}$ & $d^{M_{0}}$ & $d^{N_{0}}$ & $c^{V_{0}}$ & $c^{N_{0}}$ \\
\hline \multirow{3}{*}{1} & 0 & 2.603 & 4.294 & \multirow{3}{*}{19.210} & \multirow{3}{*}{9.605} & 3.437 & 2.147 \\
\hline & 0.3 & 3.757 & 3.574 & & & 2.861 & 1.787 \\
\hline & 0.5 & 5.013 & 3.094 & & & 2.477 & 1.547 \\
\hline \multirow{3}{*}{0.8} & 0 & 2.458 & 4.002 & \multirow{3}{*}{15.714} & \multirow{3}{*}{7.857} & 3.595 & 2.001 \\
\hline & 0.3 & 3.499 & 3.354 & & & 3.013 & 1.677 \\
\hline & 0.5 & 4.610 & 2.922 & & & 2.625 & 1.461 \\
\hline \multirow{3}{*}{0.6} & 0 & 1.988 & 4.060 & \multirow{3}{*}{12.904} & \multirow{3}{*}{6.452} & 4.264 & 2.030 \\
\hline & 0.3 & 2.699 & 3.484 & & & 3.659 & 1.742 \\
\hline & 0.5 & 3.409 & 3.100 & & & 3.256 & 1.550 \\
\hline \multirow{3}{*}{0.4} & 0 & 1.309 & 4.692 & \multirow{3}{*}{10.787} & \multirow{3}{*}{5.394} & 6.148 & 2.346 \\
\hline & 0.3 & 1.643 & 4.188 & & & 5.488 & 2.094 \\
\hline & 0.5 & 1.942 & 3.852 & & & 5.048 & 1.926 \\
\hline
\end{tabular}

Table 4: Numerical results according to the Timoshenko beam based model and $d^{M_{0}}$ based matching for orthotropic materials with $\rho=1$ and $\lambda=0.5$. 


\begin{tabular}{|c|c|c|c|c|c|c|c|}
\hline$\eta$ & $v$ & $k_{w}$ & $d^{V_{0}}$ & $d^{M_{0}}$ & $d^{N_{0}}$ & $c^{V_{0}}$ & $c^{N_{0}}$ \\
\hline \multirow{3}{*}{1} & 0 & 2.604 & 4.294 & \multirow{3}{*}{19.210} & \multirow{3}{*}{9.605} & 3.437 & 2.147 \\
\hline & 0.3 & 3.759 & 3.574 & & & 2.860 & 1.787 \\
\hline & 0.5 & 5.016 & 3.094 & & & 2.476 & 1.547 \\
\hline \multirow{3}{*}{0.8} & 0 & 2.601 & 3.891 & \multirow{3}{*}{15.589} & \multirow{3}{*}{7.795} & 3.467 & 1.945 \\
\hline & 0.3 & 3.744 & 3.243 & & & 2.889 & 1.621 \\
\hline & 0.5 & 4.984 & 2.811 & & & 2.504 & 1.405 \\
\hline \multirow{3}{*}{0.6} & 0 & 2.590 & 3.557 & \multirow{3}{*}{12.415} & \multirow{3}{*}{6.208} & 3.594 & 1.778 \\
\hline & 0.3 & 3.688 & 2.981 & & & 3.012 & 1.490 \\
\hline & 0.5 & 4.859 & 2.597 & & & 2.624 & 1.298 \\
\hline \multirow{3}{*}{0.4} & 0 & 2.598 & 3.331 & \multirow{3}{*}{9.693} & \multirow{3}{*}{4.846} & 3.921 & 1.665 \\
\hline & 0.3 & 3.606 & 2.826 & & & 3.328 & 1.413 \\
\hline & 0.5 & 4.465 & 2.490 & & & 2.932 & 1.245 \\
\hline
\end{tabular}

Table 5: Numerical results according to the Timoshenko beam based model and $d^{V_{0}}$ based matching for orthotropic materials with $\rho=1$ and $\lambda=0.5$.

\begin{tabular}{|c|c|c|c|c|c|c|c|}
\hline$\eta$ & $v$ & $k_{w}$ & $d^{V_{0}}$ & $d^{M_{0}}$ & $d^{N_{0}}$ & $c^{V_{0}}$ & $c^{N_{0}}$ \\
\hline \multirow{3}{*}{1} & 0 & 2.638 & 4.266 & \multirow{3}{*}{19.175} & \multirow{3}{*}{9.587} & 3.408 & 2.133 \\
\hline & 0.3 & 3.818 & 3.546 & & & 2.833 & 1.773 \\
\hline & 0.5 & 5.107 & 3.066 & & & 2.449 & 1.533 \\
\hline \multirow{3}{*}{0.8} & 0 & 3.864 & 3.192 & \multirow{3}{*}{14.784} & \multirow{3}{*}{7.392} & 2.697 & 1.596 \\
\hline & 0.3 & 6.084 & 2.544 & & & 2.149 & 1.272 \\
\hline & 0.5 & 8.828 & 2.112 & & & 1.784 & 1.056 \\
\hline \multirow{3}{*}{0.6} & 0 & 6.345 & 2.272 & \multirow{3}{*}{11.072} & \multirow{3}{*}{5.536} & 2.047 & 1.136 \\
\hline & 0.3 & 11.386 & 1.696 & & & 1.528 & 0.848 \\
\hline & 0.5 & 19.023 & 1.312 & & & 1.183 & 0.656 \\
\hline \multirow{3}{*}{0.4} & 0 & 11.966 & 1.552 & \multirow{3}{*}{8.041} & \multirow{3}{*}{4.021} & 1.516 & 0.776 \\
\hline & 0.3 & 26.246 & 1.048 & & & 1.023 & 0.524 \\
\hline & 0.5 & 56.875 & 0.712 & & & 0.695 & 0.356 \\
\hline
\end{tabular}

Table 6: Numerical results according to the Timoshenko beam based model and $d^{N_{0}}$ based matching for orthotropic materials with $\rho=1$ and $\lambda=0.5$.

Matching the compliance coefficients using the Euler-Bernoulli beam based model

With reference to the simplified model according to the Euler-Bernoulli beam theory, for matching based on the root rotation compliance coefficient $d_{E B}^{M_{0}}$ under moment, the use of the first of Eqn. (25b) leads to the following expression

$$
k_{w}^{M_{0} E B}=\frac{54(1+\eta)^{10}}{\eta \lambda\left(a_{1}^{M_{0}}-a_{2}^{M_{0}}\right)^{4}}
$$

where $d_{E B}^{M_{0}}=a_{1}^{M_{0}}-a_{2}^{M_{0}}$ has been enforced. Substituting Eqn. (B.2) into Eqns. (25a) and (25b) gives the remaining compliance coefficients. Similarly, for matching based on the root rotation compliance coefficient $d_{E B}^{N_{0}}$ under normal force, from the second of Eqn. (25b) we derive

$$
k_{w}^{N_{0} E B}=\frac{27(1+\eta)^{10}}{8 \eta \lambda\left(a_{1}^{N_{0}}-a_{2}^{N_{0}}\right)^{4}}
$$




\begin{tabular}{|c|c|c|c|c|c|c|}
\hline$\eta$ & $k_{w}^{E B}$ & $d_{E B}^{V_{0}}$ & $d_{E B}^{M_{0}}$ & $d_{E B}^{N_{0}}$ & $c_{E B}^{V_{0}}$ & $c_{E B}^{N_{0}}$ \\
\hline 1 & 0.812 & 5.436 & 16.154 & 8.077 & 3.659 & 2.718 \\
\hline 0.8 & 0.790 & 4.990 & 13.214 & 6.607 & 3.769 & 2.495 \\
\hline 0.6 & 0.714 & 4.791 & 10.851 & 5.426 & 4.231 & 2.396 \\
\hline 0.4 & 0.577 & 4.998 & 9.071 & 4.536 & 5.507 & 2.499 \\
\hline
\end{tabular}

Table 7: Numerical results according to the Euler-Bernoulli beam based model and $d_{E B}^{M_{0}}$ based matching for orthotropic materials with $\rho=\lambda=1$.

\begin{tabular}{|c|c|c|c|c|c|c|}
\hline$\eta$ & $k_{w}^{E B}$ & $d_{E B}^{V_{0}}$ & $d_{E B}^{M_{0}}$ & $d_{E B}^{N_{0}}$ & $c_{E B}^{V_{0}}$ & $c_{E B}^{N_{0}}$ \\
\hline 1 & 0.812 & 5.436 & 16.153 & 8.077 & 3.659 & 2.718 \\
\hline 0.8 & 0.816 & 4.911 & 13.109 & 6.555 & 3.680 & 2.456 \\
\hline 0.6 & 0.833 & 4.435 & 10.440 & 5.220 & 3.768 & 2.218 \\
\hline 0.4 & 0.885 & 4.035 & 8.151 & 4.075 & 3.995 & 2.018 \\
\hline
\end{tabular}

Table 8: Numerical results according to the Euler-Bernoulli beam based model and $d_{E B}^{V_{0}}$ based matching for orthotropic materials with $\rho=\lambda=1$.

The third case of matching based on the root rotation compliance coefficient $d_{E B}^{V_{0}}$ under transverse force deserves more attention. Neglecting the effects of shear deformations in fracture specimens approximated as Euler-Bernoulli beams leads to unacceptable underestimates of the energy release rate. In order to overcome this limit, as suggested in [10], it appears more suitable to base the matching of $d_{E B}^{V_{0}}$ on a modified root rotation coefficient obtained by modifying the exact $2 \mathrm{D}$ results $a_{1}^{V_{0}}-a_{2}^{V_{0}}$ to account for the effects of the shear deformations so that they are correctly modelled in the energy release rate. This is done by equating

$$
d_{E B}^{V_{0}}=a_{1}^{V_{0} E B}-a_{2}^{V_{0} E B}=\left(a_{1}^{V_{0}}-a_{2}^{V_{0}}\right)+(1+\eta) \kappa_{s}^{-1}\left(\frac{\rho}{\sqrt{\lambda}}+v_{\chi \chi}\right)
$$

where the second term on the right hand side of Eqn. (B.4) comes directly from the expression of the energy release rate defined in Eqn. (6) in [3] in terms of modified crack tip stress resultants. For symmetric specimens this is equivalent to perform matching on the energy release rate. Combining Eqn. (B.4) and the second of Eqn. (25a) gives

$$
k_{w}^{V_{0} E B}=\frac{3(1+\eta)^{4}}{2 \eta \lambda\left(a_{1}^{V_{0} E B}-a_{2}^{V_{0} E B}\right)^{2}} .
$$

This modification implies that, in the case for $\eta=1$, the correction factors obtained by matching any of the three root compliance coefficients coincide.

The rescaling laws presented in [3] for the root compliance coefficients $d^{K}=a_{1}^{K}-a_{2}^{K}$ show that the correction factors obtained using Eqns. (B.3-5) are independent of $\lambda$. The root compliance coefficients in Eqn. (25) for $\lambda \neq 1$ can then be easily obtained by rescaling the values corresponding to $\lambda=1$ by $\lambda^{-1 / 4}$ for $d_{E B}^{M_{0}}$ and $d_{E B}^{N_{0}}$, by $\lambda^{-1 / 2}$ for $d_{E B}^{V_{0}}=c_{E B}^{M_{0}}$ and $c_{E B}^{N_{0}}$ and by $\lambda^{-3 / 4}$ for $c_{E B}^{V_{0}}$. Tabs. 7 to 9 report results for $\rho=1$ and $\lambda=1$.

\begin{tabular}{|c|c|c|c|c|c|c|}
\hline$\eta$ & $k_{w}^{E B}$ & $d_{E B}^{V_{0}}$ & $d_{E B}^{M_{0}}$ & $d_{E B}^{N_{0}}$ & $c_{E B}^{V_{0}}$ & $c_{E B}^{N_{0}}$ \\
\hline 1 & 0.818 & 5.416 & 16.124 & 8.062 & 3.639 & 2.708 \\
\hline 0.8 & 1.009 & 4.417 & 12.432 & 6.216 & 3.138 & 2.208 \\
\hline 0.6 & 1.317 & 3.527 & 9.310 & 4.655 & 2.672 & 1.763 \\
\hline 0.4 & 1.868 & 2.777 & 6.762 & 3.381 & 2.281 & 1.389 \\
\hline
\end{tabular}

Table 9: Numerical results according to the Euler-Bernoulli beam based model and $d_{E B}^{N_{0}}$ based matching for orthotropic materials with $\rho=\lambda=1$. 


\begin{tabular}{|c|c|c|c|c|c|c|c|}
\hline \multirow{2}{*}{$\lambda$} & $\eta$ & $a_{1}^{M_{0}}-a_{2}^{M_{0}}$ & $a_{1}^{N_{0}}-a_{2}^{N_{0}}$ & \multicolumn{3}{|c|}{$a_{1}^{V_{0}}-a_{2}^{V_{0}}$} & $a_{1}^{V_{0} E B}-a_{2}^{V_{0} E B}$ \\
\hline \multirow{4}{*}{1} & 1 & 16.154 & 8.062 & 3.036 & 2.316 & 1.836 & 5.436 \\
\cline { 2 - 8 } & 0.8 & 13.214 & 6.216 & 2.751 & 2.103 & 1.671 & 4.911 \\
\cline { 2 - 8 } & 0.6 & 10.851 & 4.655 & 2.515 & 1.939 & 1.555 & 4.435 \\
\cline { 2 - 8 } & 0.4 & 9.071 & 3.381 & 2.355 & 1.851 & 1.515 & 4.035 \\
\hline \multirow{3}{*}{0.5} & 1 & 19.210 & 9.587 & 4.294 & 3.574 & 3.094 & 7.688 \\
\cline { 2 - 8 } & 0.8 & 15.714 & 7.392 & 3.891 & 3.243 & 2.811 & 6.945 \\
\cline { 2 - 8 } & 0.6 & 12.904 & 5.536 & 3.557 & 2.981 & 2.597 & 6.272 \\
\cline { 2 - 8 } & 0.4 & 10.787 & 4.021 & 3.330 & 2.826 & 2.490 & 5.706 \\
\hline
\end{tabular}

Table 10: Numerical values of the root rotations calculated from [3] and used for matching for orthotropic materials with $\rho=1$ and $\lambda=0.5,1$. 